\title{
Effect of serial ultrasound scans and optimized fetal weight limits on reduction of stillbirth risk: population-based cohort study
}

\author{
Oliver Hugh ${ }^{1}$, Andre Francis ${ }^{1}$, and Jason Gardosi ${ }^{1}$ \\ ${ }^{1}$ Perinatal Institute
}

May 20, 2020

\begin{abstract}
Objective: To assess the effect of serial growth scans and optimised fetal weight limits on the risk of stillbirth in low and highrisk maternity populations. Design: Retrospective cohort study Setting: United Kingdom 2015-2020 Population: 1,572,817 singleton pregnancies cared for in maternity units that have implemented the Growth Assessment Protocol (GAP). Methods: Analysis of fully anonymised, prospectively recorded core data from 132 National Health Service institutions. Stillbirth rate and relative risk was assessed according to risk status assigned at booking. We constructed receiver operator curves (ROC) and determined area under the curve (AUC) and optimal centile points using Youden's Index. Main Outcome: Rate of stillbirth from 24 weeks gestation. Results: The overall cohort included 6569 stillbirths (rate per thousand: 4.18). The rate was higher in pregnancies that had been designated high risk (6.23) than low risk (3.61; RR 1.7, CI 1.6-1.8). High risk pregnancies that did not receive monitoring by serial ultrasound had a stillbirth rate that was more than twice as high than those that did get serial scans as per protocol (11.94 vs 5.64). The optimal centile point for predicting stillbirth was 11.3 for the overall cohort (sensitivity 36.8, specificity 84.1 and AUC 62.3\%), 15.2 for low risk pregnancies (sensitivity 35.1, specificity 84.6, AUC 61.3\%) and 2.7 for high risk pregnancies (sensitivity 34.2, specificity 88.9, AUC 61.2\% ). Conclusions: Serial third trimester growth scans can halve stillbirth risk in pregnancies designated high risk. Optimal fetal size limits for antenatal surveillance are specific to the risk status of pregnancy.
\end{abstract}

Tweetable abstract: Serial growth scans in high risk pregnancies reduce the risk of stillbirth by half

Keywords: Fetal growth; fetal weight; birthweight; small for gestational age; stillbirth risk

\section{Introduction}

Fetal growth restriction is associated with stillbirth and other adverse perinatal outcomes ${ }^{1}$. Antenatal surveillance of fetal growth is based principally on serial assessment of fundal height, and in high risk pregnancy on serial biometry of fetal size, often defined by estimated fetal weight (EFW) ${ }^{2}$. The small for gestational age (SGA) fetus represents a significantly increased stillbirth risk ${ }^{3}$ and its identification serves as a prompt for further investigations including various Doppler indices at different stages in pregnancy, which are less effective when the fetus is not SGA ${ }^{4}$. At birth, SGA is associated with hypoglycaemia and perinatal morbidity.

The conventional definition for SGA has been the $10^{\text {th }}$ centile for over 50 years ${ }^{5}$. Other limits have since been proposed and in general, lower cut-offs such as the $3^{\text {rd }}$ and $5^{\text {th }}$ centiles have been found to have a stronger association with adverse outcome ${ }^{6,7}$. A fetus with an $\mathrm{EFW}<3^{\text {rd }}$ centile is more likely to be growth restricted and should be considered for early delivery ${ }^{8}{ }^{9}$. However cases above this limit but still below the $10^{\text {th }}$ centile, when defined by customised centiles to exclude constitutional smallness, are also at increased risk $^{10,11}$.

We wanted to investigate limits for fetal growth surveillance, with stillbirth as outcome in low and high-risk 
pregnancy. The optimal centile cut-off is a compromise between two competing objectives: first, increasing sensitivity - to identify as many cases with adverse outcomes as possible; and second, increasing specificity - to reduce the number of false positives. The optimal point also depends on the standard used to determine the centile and the population being screened. We used the RCOG-recommended ${ }^{4}$ customised standard which adjusts for constitutional variation to determine the individual growth potential ${ }^{12}$ and better predicts adverse outcome than population based standards ${ }^{13,14,15,16}$ while reducing false positives ${ }^{17}$. We undertook the analysis in low vs high risk populations according to early pregnancy assessment, as defined by $\mathrm{RCOG}^{4}$ and NHSE guidelines ${ }^{8}$, and as implemented with the Growth Assessment Protocol $(\mathrm{GAP})^{18}$ in most maternity units in the UK.

\section{Methods}

\section{Data}

The dataset was derived from pregnancies managed in the UK national GAP programme which was running during the study period in a total of 132 NHS Trusts, Health Boards or Health and Social Care Trusts in England, Wales, Scotland and Northern Ireland. Information was entered into the GROW App software (version 1.2.6.1, www.gestation.net) which produces customised growth charts and birthweight centiles adjusted for each pregnancy according to early pregnancy maternal weight, height, parity and ethnic origin ${ }^{12}$. Also recorded was whether the pregnancy was considered at increased risk of growth restriction on the basis of obstetric or medical history and risk factors such as smoking or high body mass index, and whether serial third trimester ultrasound biometry was instituted according to protocol. The number of growth scans was not collected in the database but, according to routine audits within the programme, usually consisted of 3 to 4 third trimester scans from 28 weeks to delivery. Pregnancies were dated according to the routine $1^{\text {st }}$ trimester ultrasound scan. Outcomes recorded after delivery included birthweight, sex and gestational age at delivery, and whether live birth or stillbirth. Stillbirth was defined as a baby born with no signs of life from 24.0 weeks gestation. Gestational age for stillbirths was based on the age at delivery minus 2 days to adjust for the average delay in third trimester between intrauterine demise and delivery ${ }^{19}$.

There were 1,641,897 singleton pregnancies delivered $>24.0$ weeks gestation between January 2015 and January 2020. After excluding late fetal losses (725) and cases with missing or incomplete data $(68,355)$, the final study cohort consisted of 1,572,817 pregnancies. All data were fully anonymised including institution of origin, date of birth and all other maternal, new-born and pregnancy-related identifiers, and hence ethics approval was not required.

Statistical Methods To assess the association between customised GROW centiles and stillbirth, we developed receiver operator curves (ROCs) according to standard methodology ${ }^{20}$ on the overall cohort as well as the low and high risk subgroups. Risk status was recorded in 1,308,967 (83\%) of the cases, with the remainder missing mostly because the detailed data items were not contained in the version of the local electronic system in use during the study period.

Analyses were carried out using SAS software (version 9.4, SAS Institute, NC, USA). Optimal centile cut-offs were determined by balancing sensitivity and specificity using Youden's index ${ }^{21}$, and model performance was assessed according to area under the curve (AUC) analysis. Differences between pregnancy characteristics were determined using the Kruskal Wallis test for continuous variables and a Z Test between proportions. Outcome between groups was compared using relative risk (RR) with $95 \%$ confidence interval (CI).

\section{Results}

Characteristics of the cohort

Table 1 describes the study cohort of 1,572,817 births, of which 6,569 were stillborn (rate 4.18/1000).

Of the 1,308,967 cases where the results of early pregnancy risk assessment were recorded, 990,199 were designated low risk and had a stillbirth rate (per thousand) of 3.61 (Table 2). Cases where risk status was not recorded had a similar stillbirth rate $(3.82, \mathrm{P}=0.11)$. 


\section{Serial scans and stillbirth risk}

The 318,768 (24.4\%) pregnancies that were recorded as high risk had a shorter median length of pregnancy (273 vs 279 days, $\mathrm{p}<0.01$ ) and a $70 \%$ higher rate of stillbirth compared to pregnancies designated low risk (Table 2). In this high-risk group, 289,285 pregnancies (90.8\%) were monitored with serial scans according to protocol, and had a stillbirth rate of 5.64 (RR compared to low risk: 1.6, CI 1.5-1.7). Pregnancies which did not receive serial scans had a stillbirth rate that was more than twice as high as those that did have serial scans: 11.94 (RR compared to low risk: 3.3, CI 3.0-3.7).

\section{ROC curves to determine optimal points}

The ROC curves for the overall and low and high risk cohorts are shown in Figures 1-3 and their respective area under the curve (AUC) and diagnostic accuracy is summarised in Table 3. The overall cohort had an AUC of $62.3 \%$ with optimal centile 11.3 , with a sensitivity of $36.8 \%$ and specificity of $84.1 \%$ for detection of stillbirth. For the low-risk cohort, the AUC was $61.3 \%$ with optimal point 15.2, sensitivity $35.1 \%$ and specificity $84.6 \%$, while for the high risk cohort the AUC was $61.2 \%$, optimal centile point 2.7 , sensitivity $34.2 \%$ and specificity $88.9 \%$ (Table 3 ). With false positive rate fixed at $10 \%$ i.e. specificity $90 \%$, the sensitivity was - overall cohort: $30.2 \%$ (cut-off point 6.6 ), low risk: $29.1 \%$ (10.1) and high risk $33.0 \%$ (2.3).

\section{Discussion}

\section{Main findings}

This is to our knowledge the first determination of the best performing customised fetal weight limits for the prediction of stillbirth. Previously we calculated ROC curves for customised fetal weight centiles in a smaller Dutch cohort ${ }^{22}$ with adverse outcomes defined as operative delivery or admission to neonatal intensive care. Here, we were able to use a large database from a national programme to analyse stillbirth as a rarer, harder outcome measure in different risk groups.

While the actual AUCs were relatively modest, ranging from $61.2 \%$ to $62.3 \%$, this is not surprising as restricted growth leading to SGA is not the only factor associated with, or causal for, stillbirth ${ }^{23}$. It is consistent with AUC values reported recently from datasets with similar amounts of information available at booking. ${ }^{24}$

\section{Strengths and limitations}

The strengths of our study include the use of a large database derived from routine, prospective data entry in a national programme, and the application of a customised standard which adjusts for constitutional variation and thereby better reflects the fetus' growth potential ${ }^{25}$. In a proportion of cases the early pregnancy risk assessment was not recorded, as the data field was not contained in the electronic maternity record. However, the similar stillbirth rate in this 'missing' group suggests that this did not introduce a systematic bias.

We had no ultrasound measurements recorded in this database and therefore the trajectory of growth, expressed as velocity or growth rate, could not be calculated. While serial scans can provide useful information about growth rate ${ }^{26}$, it has also been argued that it adds little to the effectiveness of the last scan to predict adverse outcome ${ }^{27-30}$. The use of birthweight-based centiles could be considered a weakness of the study, as the objective was to predict risk of stillbirth based on fetal rather than neonatal weight. However, it can also be considered a strength, as 1. it allowed comparison between subgroups (the low risk group having had no clinical indication to undergo ultrasound investigations); 2 . There was no need to adjust case by case for the respective scan-to-delivery interval; and 3. it is appropriate to infer fetal weight from newborn weight as gold standard, as estimated fetal weight is prone to systematic and random measurement error ${ }^{31}$. It was possible to apply this method as the customised GROW chart provides the same, contiguous standard for fetal and neonatal weight.

\section{Interpretation}


The results of our analyses have immediate clinical utility for antenatal surveillance of fetal growth. Firstly, the $11^{\text {th }}$ centile as a threshold for the whole population, regardless of risk assessment, confirms that the conventional $10^{\text {th }}$ centile for SGA remains a useful general standard. The $15^{\text {th }}$ centile as best cut-off to assess stillbirth risk in a low risk population (Figure 2) is a new finding and may be a useful limit for fundal height measurements but requires further investigation. A similar cut-off has been proposed for the assessment of risk of neonatal death ${ }^{32}$.

Secondly, the observed optimal threshold of the $2.7^{\text {th }}$ centile in high risk pregnancies would confirm the $3^{\text {rd }}$ centile line as an appropriate cut-off to indicate the presence of fetal growth restriction, as proposed in the NHS England guidelines ${ }^{33}$ and the new GAP care pathway ${ }^{34}$. The recommendation is that a fetus with a weight below $3^{\text {rd }}$ centile should be considered for delivery by 37 weeks, regardless of the results of umbilical artery Doppler investigation because of its limited effectiveness for the assessment of growth status late in third trimester ${ }^{9}$. Fetuses with a weight between the $3^{\text {rd }}$ and $10^{\text {th }}$ centile are also at risk, but delivery can be delayed until 39 weeks if Doppler indices more suitable for assessment at term (uterine artery and middle cerebral artery) remain normal. ${ }^{9,33,34}$

\section{Conclusion}

Our study highlighted the importance of early pregnancy assessment of stillbirth risk and adherence to a growth surveillance protocol in high risk pregnancies. The risk of stillbirth can be halved by serial scanning during the third trimester. Appropriate cut-off points for fetal weight assessment vary according to risk status, ranging from $15^{\text {th }}$ centile in low risk to $3^{\text {rd }}$ centile in high risk pregnancies.

Disclosure of interests All authors are employed by the Perinatal Institute, a not for profit social enterprise that provides training and software tools including GROW charts as part of the Growth Assessment Protocol referred to in this study.

Contribution to authorship All authors contributed to the idea of the study and its design and data analysis, and jointly drafted, revised and agreed the manuscript.

Ethics All data were recorded as part of routine care, retrospectively collected and fully anonymised, and hence ethics committee approval was not required.

Funding - None

\section{Acknowledgements - None}

\section{References}

1. Kady S, Gardosi J. Perinatal mortality and fetal growth restriction. Best Pract Res Clin Obstet Gynaecol. 2004 Jun;18(3):397-410. www.doi.org/10.1016/j.bpobgyn.2004.02

2. Figueras F, Gardosi J. Intrauterine growth restriction: new concepts in antenatal surveillance, diagnosis, and management. Am J Obstet Gynecol. 2011 Apr;204(4):288-300. www.doi.org/10.1016/j.ajog.2010.08.055

3. Gardosi J, Madurasinghe V, Williams M, Malik A, Francis A. Maternal and fetal risk factors for stillbirth: population based study. BMJ . 2013 Jan 24;346(3). www.doi.org/10.1136/bmj.f108

4. Royal College of Obstetricians and Gynaecologists. The Investigation and Management of the Small for Gestational Age Fetus. Green Top Guideline No 31. 2013 www.rcog.org.uk/en/guidelines-researchservices/guidelines/gtg31/

5. Battaglia FC, Lubchenco LO. A practical classification of newborn infants by weight and gestational age. J Pediatr. 1967 Aug 1;71(2):159-63. www.doi.org/10.1016/s0022-3476(67)80066-0

6. Pilliod RA, Cheng YW, Snowden JM, Doss AE, Caughey AB. The risk of intrauterine fetal death in the small-for-gestational-age fetus. Am J Obstet Gynecol. 2012 Oct;207(4):318.e1-318.e6. www.doi.org/10.1016/j.ajog.2012.06.039

7. Gordijn SJ, Beune IM, Thilaganathan B, Papageorghiou A, Baschat AA, Baker PN, et al. Consensus definition of fetal growth restriction: a Delphi procedure: Consensus definition of FGR. Ultrasound 
Obstet Gynecol. 2016 Sep;48(3):333-9. www.doi.org/10.1002/uog.15884

8. NHS England. Saving Babies Lives: A care bundle for reducing stillbirth. 2016. www.england.nhs.uk/wp-content/uploads/2016/03/saving-babies-lives-car-bundl.pdf

9. Figueras F, Gratacos E. An integrated approach to fetal growth restriction. Best Pract Res Clin Obstet Gynaecol. 2017 Jan 1;38:48-58. www.doi.org/10.1016/j.bpobgyn.2016.10.006

10. Clausson B, Gardosi J, Francis A, Cnattingius S. Perinatal outcome in SGA births defined by customised versus population-based birthweight standards. Br J Obstet Gynaecol. 2001;108(8):830-834. www.doi.org/10.1111/j.1471-0528.2001.00205.x

11. Williams M, Turner S, Hugh O, Gardosi J. Reduction of small-for-gestational-age stillbirths at term in the GAP programme 2015-2018. Br J Obstet Gynaecol. 2019;126(S2):53. www.bit.ly/2YxH7a5

12. Gardosi J, Mongelli M, Wilcox M, Chang A. An adjustable fetal weight standard. Ultrasound Obstet Gynecol. 1995 Sep 1;6(3):168-74. www.doi.org/10.1046/j.1469-0705.1995.06030168.x

13. Gardosi J, Clausson B, Francis A. The value of customised centiles in assessing perinatal mortality risk associated with parity and maternal size: Value of customising centiles for parity and maternal size. Br J Obstet Gynaecol. 2009 Sep;116(10):1356-63. www.doi.org/10.1111/j.1471-0528.2009.02245.x

14. Gardosi J, Francis A. Adverse pregnancy outcome and association with small for gestational age birthweight by customised and population-based percentiles. Am J Obstet Gynecol. 2009 Jul;201(1):28.e128.e8. www.doi.org/10.1016/j.ajog.2009.04.034

15. Figueras F, Figueras J, Meler E, Eixarch E, Coll O, et al. Customised birthweight standards accurately predict perinatal morbidity. Arch Dis Child - Fetal Neonatal Ed. 2007 Jan 24;92(4):F277-80. www.dx.doi.org/10.1136\%2Fadc.2006.108621

16. McCowan LM, Harding JE, Stewart AW. Customised birthweight centiles predict SGA pregnancies with perinatal morbidity. Br J Obstet Gynaecol. 2005;112(8):1026-1033. www.doi.org/10.1111/j.14710528.2005.00656.x

17. Mongelli M, Gardosi J. Reduction of false-positive diagnosis of fetal growth restriction by application of customised fetal growth standards. Obstet Gynecol. 1996;88(5):844-848. www.doi.org/10.1111/j.14710528.2005.00656.x

18. Clifford S, Giddings S, Southam M, Williams M, Gardosi J. The Growth Assessment Protocol: a national programme to improve patient safety in maternity care. MIDIRS Midwifery Dig. 2013;23(4):51623 https://tinyurl.com/yddry7ap

19. Gardosi J, Mul T, Mongelli M, Fagan D. Analysis of birthweight and gestational age in antepartum stillbirths. Br J Obstet Gynaecol. 1998;105(5):524-530. www.doi.org/10.1111/j.1471-0528.1998.tb10153.x

20. Metz CE. Basic principles of ROC analysis. Semin Nucl Med. 1978 Oct;8(4):283-98. www.doi.org/10.1016/s0001-2998(78)80014-2

21. Youden WJ. Index for rating diagnostic tests. Cancer. 1950;3(1):32-5. www.doi.org/10.1002/10970142(1950)3:1\%3C32::aid-cncr2820030106\%3E3.0.co;2-3

22. De Jong CLD, Francis A, Van Geijn HP, Gardosi J. Customised fetal weight limits for antenatal detection of fetal growth restriction. Ultrasound Obstet Gynecol. 2000;15(1):36-40. www.doi.org/10.1046/j.1469-0705.2000.00001.x

23. Allanson ER, Tunçalp Ö, Gardosi J, Pattinson RC, Francis A, Vogel JP, et al. The WHO application of ICD-10 to deaths during the perinatal period (ICD-PM): results from pilot database testing in South Africa and United Kingdom. BJOG Int J Obstet Gynaecol. 2016;123(12):2019-2028. www.doi.org/10.1111/1471-0528.14244

24. Malacova E, Tippaya S, Bailey HD, Chai K, Farrant BM, Gebremedhin AT, et al. Stillbirth risk prediction using machine learning for a large cohort of births from Western Australia, 1980-2015. Sci Rep. 2020 Mar 24;10(1):1-8. www.doi.org/10.1038/s41598-020-62210-9

25. Gardosi J, Francis A, Turner S, Williams M. Customised growth charts: rationale, validation and clinical benefits. Am J Obstet Gynecol. 2018;218(2). www.doi.org/10.1016/j.ajog.2017.12.011

26. Francis A, Hugh O, Gradosi J. Slow growth defined by customised growth trajectory and adverse perinatal outcome. In: 8th International Conference on Fetal Growth . Berlin: Abstract O4.3; 2019. www.bit.ly/2KZHJBa 
27. Iraola A, González I, Eixarch E, Meler E, Illa M, Gardosi J, et al. Prediction of adverse perinatal outcome at term in small for gestational age fetuses: comparison of growth velocity vs. customised assessment. J Perinat Med. 2008 Nov 1;36(6):531-5. www.doi.org/10.1515/JPM.2008.100

28. Tarca AL, Hernandez-Andrade E, Ahn H, Garcia M, Xu Z, Korzeniewski SJ, et al. Single and Serial Fetal Biometry to Detect Preterm and Term Small- and Large-forGestational-Age Neonates: A Longitudinal Cohort Study. PloS One. 2016 Nov 1;11(11):e0164161. www.doi.org/10.1371/journal.pone.0164161

29. Caradeux J, Eixarch E, Mazarico E, Basuki TR, Gratacós E, Figueras F. Second- to third-trimester longitudinal growth assessment for prediction of small-for-gestational age and late fetal growth restriction. Ultrasound Obstet Gynecol. 2018;51(2):219-24. https://doi.org/10.1002/uog.17471

30. Ciobanu A, Formuso C, Syngelaki A, Akolekar R, Nicolaides KH. Prediction of small-for-gestationalage neonates at 35-37 weeks' gestation: contribution of maternal factors and growth velocity between 20 and 36 weeks: Third trimester screening for SGA. Ultrasound Obstet Gynecol. 2019 Feb; 3(4):488-495. www.doi.org/10.1002/uog.20243

31. Dudley N. A review of ultrasound fetal weight estimation in the early prediction of low birthweight. Ultrasound. 2013 Nov;21(4):181-6. www.doi.org/10.1177\%2F1742271X13505259

32. Xu H, Simonet F, Luo Z-C. Optimal birth weight percentile cut-offs in defining small- or large-forgestational-age. Acta Paediatr. 2010;99(4):550-5. www.doi.org/10.1111/j.1651-2227.2009.01674.x

33. NHS England. Saving Babies' Lives v.2: A care bundle for reducing perinatal mortality. NHS England. 2019. www.england.nhs.uk/publication/saving-babies-lives-version-two-a-care-bundlefor-reducing-perinatal-mortality/

34. Perinatal Institute. GAP Care Pathway v2. 2020. www.perinatal.org.uk/GAP/GAP_Care_Pathway_v2.pdf

\section{List of Tables and Figures}

Table 1: Characteristics of study cohort $(n=1,572,817)$

Table 2: Pregnancy outcome according to risk status $(\mathrm{n}=1,572,817)$

Table 3: Result of ROC analysis ( $\mathrm{n}=1,308,967$ with risk status recorded)

Figure 1: ROC for entire cohort ( $\mathrm{n}=1,572,817$; Optimal point 11.3; AUC 62.3)

Figure 2: ROC for low risk group $(\mathrm{n}=990,199$; Optimal point 15.2; AUC 61.3)

Figure 3: ROC for high risk group ( $\mathrm{n}=318,768$; Optimal point 2.7; AUC 61.2)

Table 1 - Characteristics of study cohort $(n=1,572,817)$

\begin{tabular}{lllll}
\hline & Mean & SD & Median & IQR \\
\hline Maternal Height $(\mathrm{cm})$ & 164.3 & 6.6 & 164.0 & $160.0-169.0$ \\
Maternal Weight $(\mathrm{kg})$ & 71.4 & 16.9 & 68.0 & $60.0-80.0$ \\
Body Mass Index $\left(\mathrm{kg} / \mathrm{m}^{2}\right)$ & 26.5 & 5.9 & 25.2 & $22.2-29.4$ \\
& $\mathrm{n}$ & $\%$ & & \\
Parity & & & & \\
0 & 658,800 & 41.9 & & \\
1 & 544,685 & 34.6 & & \\
2 & 226,028 & 14.4 & & \\
$3+$ & 143,304 & 9.1 & & \\
BMI $>35 \mathrm{~kg} / \mathrm{m}^{2}$ & 144,035 & 9.2 & & \\
Ethnicity & & & & \\
British European & $1,110,717$ & 70.6 & & \\
East European & 100,589 & 6.4 & & \\
& & &
\end{tabular}




\begin{tabular}{lllll}
\hline & Mean & SD & Median & IQR \\
\hline Other European & 36,953 & 2.3 & & \\
South Asian & 136,634 & 8.7 & & \\
Other Asian & 34,586 & 2.2 & & \\
African & 45,897 & 2.9 & & \\
Mixed & 15,007 & 1.0 & & \\
Other & 92,434 & 5.9 & & \\
Preterm (<37 weeks) & 101,336 & 6.4 & & \\
(<34 weeks) & 23,253 & 1.5 & & \\
Stillbirth & 6569 & 4.18 & $/ 1000$ & \\
& Mean & SD & Median & IQR \\
Gestation (days) & 275.8 & 12.9 & 277 & $270-284$ \\
Birthweight (grams) & 3352 & 565 & 3375 & $3025-3720$ \\
Customised Centile & 45.2 & 29.7 & 42.6 & $18.8-70.3$ \\
\hline
\end{tabular}

Table 2 - Pregnancy outcome according to risk status $(\mathrm{n}=1,572,817)$

\begin{tabular}{lllllllll}
\hline & Births & Births & Gestation & Gestation & Stillbirths & Stillbirths & \\
& $\mathrm{N}$ & $\%$ & Median (days) & $\%<37$ weeks & $\mathrm{n}$ & $/ 1000$ & $\mathrm{RR}$ & $95 \%$ CI \\
Low Risk & 990,199 & 75.6 & 279.0 & 4.4 & 3,575 & 3.61 & 1.0 & - \\
High Risk & 318,768 & 24.4 & 273.0 & 12.6 & 1,985 & 6.23 & 1.7 & $1.6-1.8$ \\
- with serial scans & 289,285 & 90.8 & 273.0 & 12.5 & 1,633 & 5.64 & 1.6 & $1.5-1.7$ \\
- without serial scans & 29,483 & 9.2 & 274.0 & 13.4 & 352 & 11.94 & 3.3 & $3.0-3.7$ \\
Total Recorded & $1,308,967$ & 100.0 & 277.0 & 6.4 & 5,560 & 4.25 & & \\
Not recorded & 263,850 & 16.8 & 278.0 & 6.6 & 1009 & 3.82 & & \\
\hline
\end{tabular}

RR, Relative Risk; CI, Confidence Interval

Table 3 - Result of ROC analysis ( $\mathrm{n}=1,308,967$ with risk status recorded)

\begin{tabular}{llllll}
\hline & Optimal centile & Proportion (\%) below cut off & Proportion (\%) below cut off & AUC \% & Sensitivity \% \\
Overall cohort & 11.3 & 16.0 & 62.3 & 62.3 & 62.3 \\
Low Risk & 15.2 & 15.5 & 61.3 & 61.3 & 61.3 \\
High Risk & 2.7 & 11.2 & 61.2 & 61.2 & 61.2 \\
\hline
\end{tabular}

ROC, Receiver Operator Curve; AUC, Area Under Curve; CI, Confidence Interval; PPV, Positive Predictive Value; NPV, Negative Predictive Value.

\section{Hosted file}

Figure 1.docx available at https://authorea.com/users/317990/articles/448005-effect-ofserial-ultrasound-scans-and-optimized-fetal-weight-limits-on-reduction-of-stillbirthrisk-population-based-cohort-study

\section{Hosted file}

Figure 2.docx available at https://authorea.com/users/317990/articles/448005-effect-ofserial-ultrasound-scans-and-optimized-fetal-weight-limits-on-reduction-of-stillbirth- 
risk-population-based-cohort-study

\section{Hosted file}

Figure 3.docx available at https://authorea.com/users/317990/articles/448005-effect-ofserial-ultrasound-scans-and-optimized-fetal-weight-limits-on-reduction-of-stillbirthrisk-population-based-cohort-study 